\title{
Management of Broken Endodontic Instrument Using C-Arm System
}

\author{
Rajkumar Balakrishnan'1, Gupta Vishesh², \\ Bhatt Akanksha ${ }^{3}$, Thind Amandeep ${ }^{4}$
}

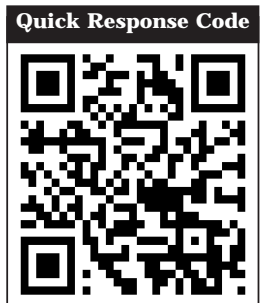

doi : $10.5866 / 2016.8 .10251$

${ }^{1}$ Professor \& HOD

${ }^{2}$ Associate Professor

${ }^{3}$ Assistant Professor

Department of Conservative Dentistry \& Endodontics, BBD College of Dental Sciences, Lucknow-227105.

${ }^{4}$ Assistant Professor

Department of Conservative Dentistry \& Endodontics, Rama Dental College Hospital \& Research Centre, Kanpur-208024.

\section{Article Info:}

Received: October 8, 2016

Review Completed: November 7, 2016

Accepted: December 9, 2016

Available Online: December, 2016 (www.nacd.in)

ONAD, 2016 All rights reserved

Email for correspondence:

dr.a.bhatt@gmail.com

\begin{abstract}
:
C-arm computed tomography is a new and innovative imaging technique. In combination with 2D fluoroscopic or radiographic imaging, information provided by 3D C-arm real time imaging can be valuable for therapy planning, guidance and outcome assessment in the field of modern endodontics. This paper reports a case on bypassing separated instrument and other one on root canal treatment, using Artis zee C-arm CT system first time in endodontic practice.
\end{abstract}

Key words: C-arm CT system, Endodontics, Separated instrument, Real time Imaging.

\section{Introduction}

Encouraging results have been achieved using the latest imaging modalities like Dental ConeBeam CT in endodontics but none of these systems can provide a three-dimensional real time imaging. Carm CT is a dynamic and innovative imaging technique that differs from conventional dental radiography which is static. ${ }^{1}$ While physicians can observetheseliveimaging events, dentists have only possibility of monitoring treatment progress is by making radiographs before and after the procedure. As a result, craniofacial surgeries, endodontic treatments and conventional dental implant placement are invariably blind procedures. So, introduction of C-arm systems to endodontic practice will be a giant leap and definitely will provide a cutting edge over existing methods because of its real time imaging advantage. In the present case report, Artis zee C-arm real time imaging system (Siemens AG, Healthcare Sector, Forchheim, 
Germany) was used for precise detection and location of the separated endodontic instrument as well as to bypass the separated instrument (Figure1).

\section{Case Presentation}

A $27 \mathrm{yr}$ old female patient reported with a chief complaint of pain in lower left back tooth. On clinical and radiographic examination, the patient was diagnosed with previously attempted incomplete endodontic treatment and presence of a separated instrument in the middle $1 / 3^{\text {rd }}$ of mesiobuccal canal of 36. In the present case, use of an operating microscope couldn't provide the in-depth visualization of separated instrument due to anatomical constrictions of the canal, hence use of C-arm was decided to achieve this. The tooth was isolated using flexi dam (Coltene Whaledent, Switzerland). A preoperative scan was obtained using C-arm system (Figure2). Access was prepared and ultrasonic tips were used to create troughing around the separated instrument .An attempt to retrieve the separated instrument with instrument retrieval system along with ultrasonic vibration was unsuccessful. So it was decided to bypass the instrument to achieve working length. Bypassing procedure consisted of wedging a \#08 K-file between the canal wall and separated instrument fragment to create a space between them. The file was gradually advanced and withdrawn repeatedly along with use of $17 \%$ EDTA solution to reach theworking length. The same was verified by real time visualization on C-arm system for 3 seconds that gave an advantage to observe the file movement, bypassing the separated instrument, eliminating the risk of perforation by the clinician during the procedure. The procedure was repeated using Endosonic files (Dentsply Maillefer, Switzerland) to widen the space created between fragment and canal wall. After widening the space till working length biomechanical preparation was started using \# 15/ .04 rotary file (Hyflex CM, Coltene Whaledent, Switzerland) along with $2.5 \%$ sodium hypochlorite irrigation (Figure3) which was verified by $\mathrm{C}$-arm system for 5 seconds that aided the clinician to observe the movement of rotary file bypassing the separated instrument. Biomechanical preparation was completed using \#25/0.6 rotary files for mesiobuccal and mesio-lingual canal and \#30/0.6 for distal canal (Hyflex CM, ColteneWhal edent, Switzerland). All the three canals were obturated with $6 \% \# 25$ and $6 \% \# 30$ single gutta percha cones (Coltene Whaledent, Switzerland) with gutta flow sealer (Coltene Whaledent, Switzerland). C-arm real system was used for real time imaging with an intermittent 3 and 5 second recording mode with the strict adherence to ALARA protocol for both the patient and operator using thyroid collars and lead apron. Post obturation image was obtained using C-arm system (F igure 4).

\section{Discussion}

On literature search in PubMed data base with the words "C-arm CT in Endodontics" , "C-arm in removal of separated endodontic instrument " and " C-arm in bypassing separated endodontic instrument " no results, "C-arm CT in Dentistry" 4 results, "C-arm Fluoroscopy in Dentistry" 6 results and in Cochrane data base none of the above words yield any results.

In-office C-arm CT has hel ped to fulfil a growing demand for minimally invasive procedures in traumatology, orthopaedics, endoscopy, paediatrics and urgent care medicine. ${ }^{2}$ Today, C-arm CT accounts for $35 \%$ of the medical imaging market. ${ }^{3}$

C-arm cone beam computed tomography (CT) is an advanced imaging capability that uses stateof-art C-arm flat-panel fluoroscopy systems to acquire and display three-dimensional (3D) images. C-arm cone-beam CT provides high and low contrast soft tissue (CT-like) images in multiple viewing planes, which constitutes a substantial improvement over conventional single-plannar digital substraction angiography (DSA) and fluoroscopy. ${ }^{4}$

To obtain 2D radiographic projection data, the C-arm performs a sweep around the patient e.g. over $200^{\circ}$. Up to several hundred images are acquired 


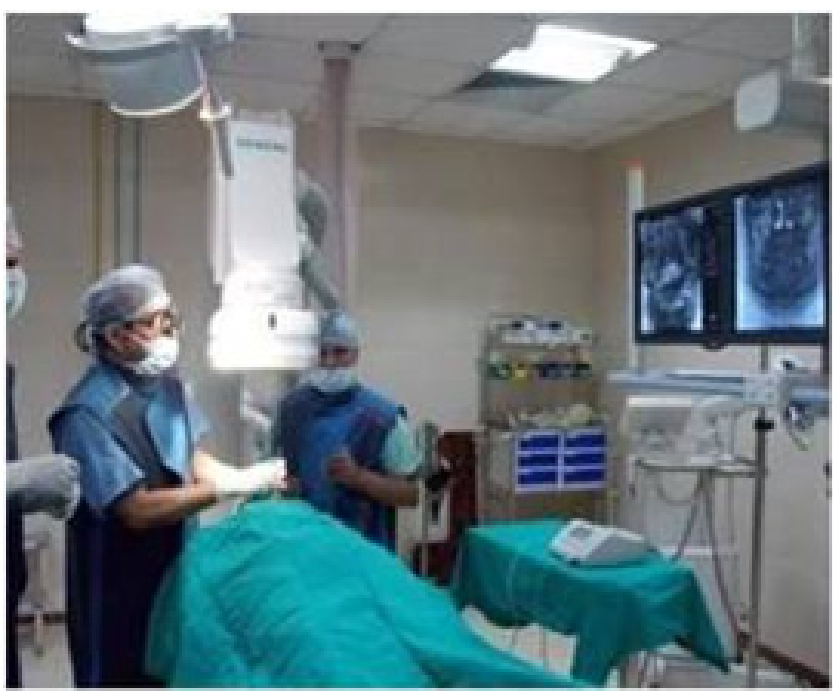

Figure 1: Endodontic procedure with Artis zee C-arm real time imaging system.

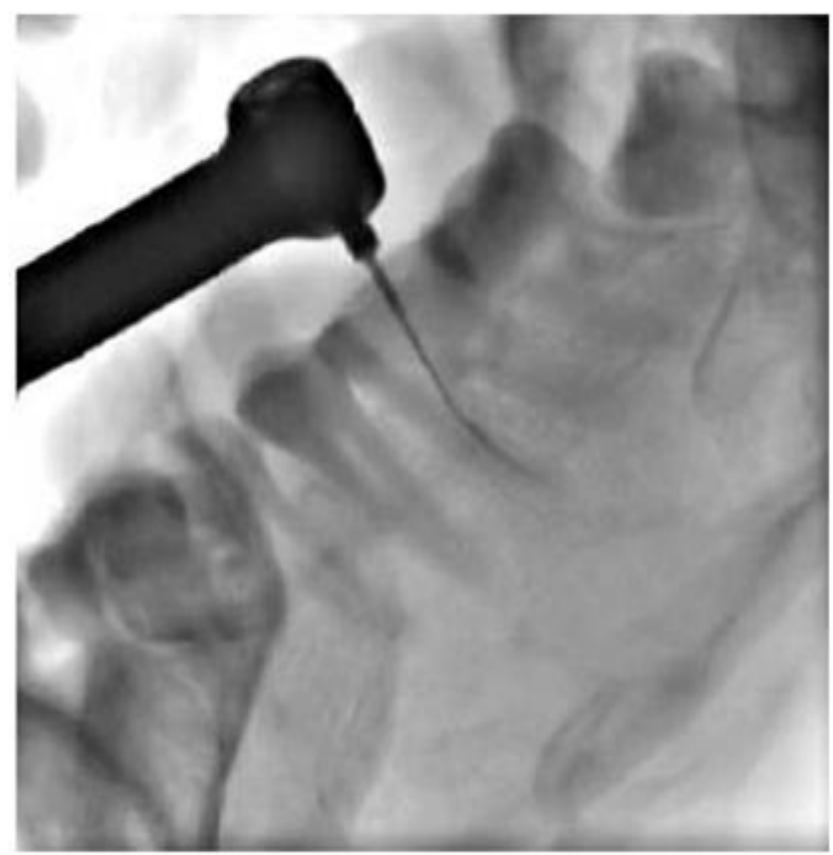

Figure 3: Biomechanical preparation in Mesiobuccal canal using $\# 15 / .04$ rotary file.

depending on the acquisition protocol selected. Reconstruction of three-dimensional voxel data sets from $2 \mathrm{D}$ raw projection data is performed using a 3D cone-beam reconstruction algorithm. Resulting voxel data sets can be visualized either as crosssectional images or as 3D data sets using different volume rendering techniques. ${ }^{1,5-7}$

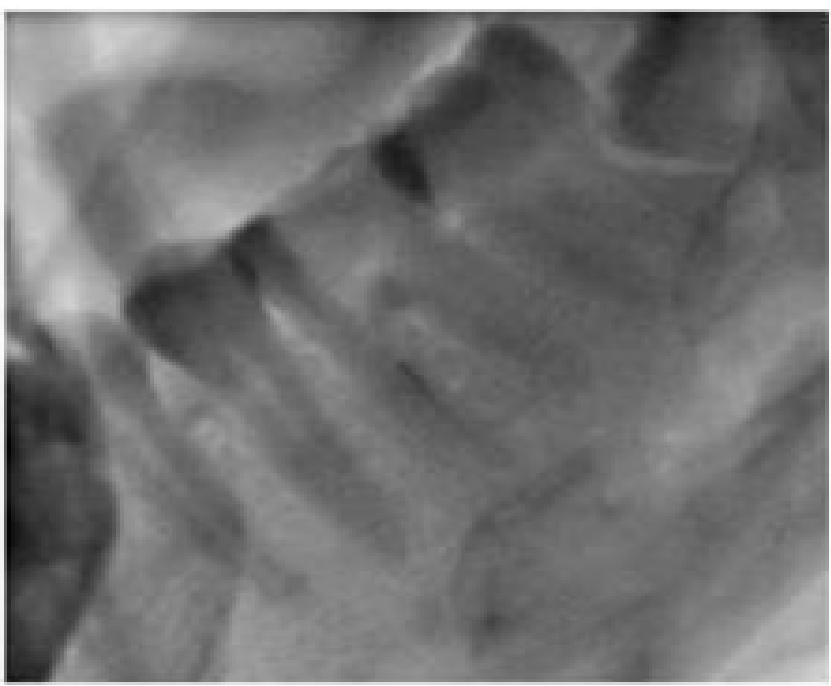

Figure 2: Preoperative I mage obtained using C-arm system.

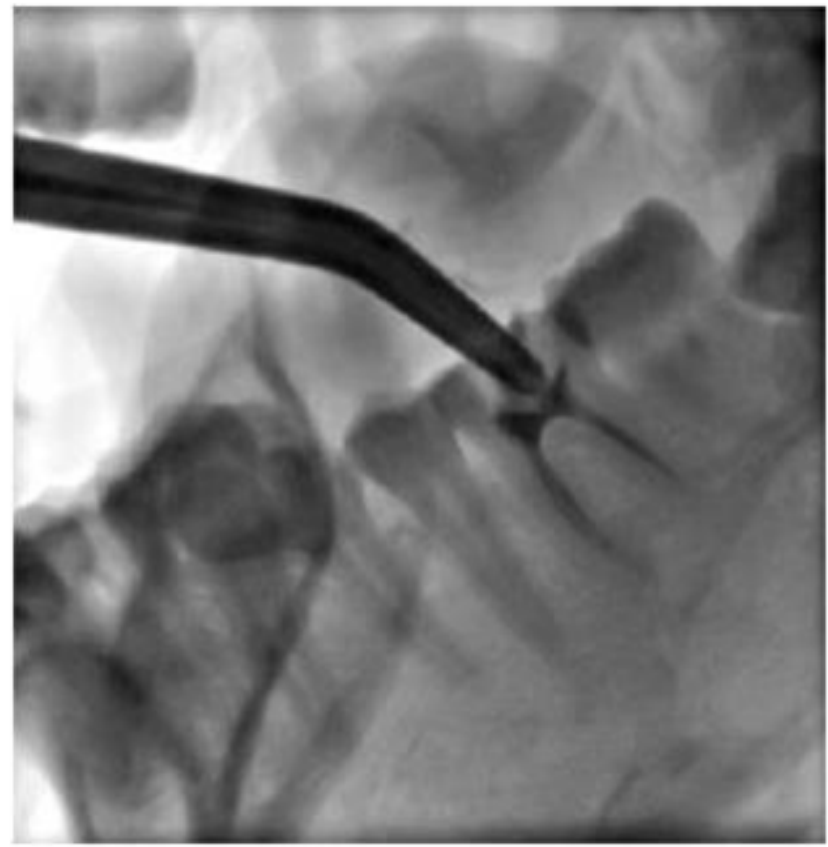

Figure 4: Post obturation I mage obtained using C-arm system.

The multi-axis stand $\mathrm{C}$ arm system facilitates greater flexibility, moreaccurate, faster movements and better patient coverage, such systems are especially well suited for minimally invasive procedures and surgery. ${ }^{1,8}$ It offers high-end applications for surgery through real time 3D imaging, high frame rates of 30 frames per second(f/ 
s), and excellent image quality at low radiation exposure, which is $60-80 \%$ less as compared to Spiral CT.9, 10 As per the manufacturers, the maximum radiation dosefor the present system used is $0.25 \mathrm{mSv}$ for 5 second exposure time.

In the present case, the real time imaging provided a 3D viewing of involved tooth intricate anatomical details, preventing chances of perforation especially while by-passing the separated instrument thus improving the quality of treatment. The limitation of this system is that it requires complete supine position of the patient and moderately higher exposure protocol and the design that suits a large volume of tissue to be scanned. So, a limited view chair side C-arm system with radiation guidelines is the need of the hour.

\section{Conclusion}

Several advanced radiography techniques have been in use in endodontics, namely CT, CBCT, TACT, micro-CT and OCT for in vitro studies. Carm CT system provides a real time imaging of the root canal system, lesions and surrounding anatomical structures, during clinical procedure, it will definitely gain lot of attention for routine endodontic practice in days to come.

\section{References}

1. Strobel N, Meissner O, Boese J BrunnerT, Heigl B, Hoheisel $\mathrm{M}$ et al. Imaging with flat-detector $\mathrm{C}$-arm systems. Multislice CT. Springer 2009 :33-51.
2. Blinov NN, Mazurov Al. An analysis of the outlook for using C-arm type mobile x-ray diagnostic apparatus. Med Tekh 2000; 5:19-23.

3. U.S. Food and Drug Administration. 21 C.F.R. Part 1020. Federal Register. Electronic Products; Performance Standard for Diagnostic X-ray Systems and Their Major Components. Final Rule 2005; 70:33998-34042.

4. Wallace MJ, Kuo MD, Glaiberman C., Binkert CA, Orth RC, Soulez G. Three-Dimensional C-arm Cone-beam CT: Applications in the interventional suite. J Vasc Inter Radiol 2008; 19(6):799-813.

5. Eisenberg RL. Radiology: An illustrated history. St Louis: Mosby-Year Book. 1992: 51-78

6. Chamberlain WE. Fluoroscopes and fluoroscopy. Radiology. 1942; 38:383-413.

7. Fahrig R, Dixon R, Payne T, Morin R, Ganguly A, and Strobel $\mathrm{N}$. Dose and image quality for a cone-beam $\mathrm{C}$-arm CT system. Med Phys 2006; 3:4541-50.

8. Zellerhoff M, Scholz B, Ruhrnschopf EP. Low contrast 3D reconstruction from $\mathrm{C}$-arm data. In: Medical imaging. Physics of medical imaging. SPIE, San Diego, CA. 2005:64655.

9. Ritter D, Orman J , Schmidgunst C. Three-dimensional soft tissue imaging with a mobile C-arm. Comput Med I maging Graphics 2007; 31:91-102.

10. Linsenmaier U, Rock C, Euler E, Wirth S, Brandl R, Kotsianos D et al. Three dimensional CT with a modified C-arm image intensifier: Feasibility. Radiology 2002; 224:286-92.

\section{Gain quick access to our journal online View our journal at www.nacd.in}

